\title{
AN EXPERIMENTAL STUDY OF \\ COUNTER-ROTATING CORES IN ELLIPTICAL GALAXIES
}

\author{
R. H. Miller, G. R. Roelofs, and B. F. Smith \\ University of Chicago \\ and \\ Theoretical Studies Branch, NASA-Ames Research Center
}

\section{Introduction}

Recent observational studies (Franx and Illingworth 1987; Jedrzejewski and Schechter 1988; Bender 1988; Illingworth and Franx 1989) have shown that some elliptical galaxies have a small region near the center that rotates in the opposite direction from the outer parts of the galaxy. Often the rotation in the central part is much faster than that in the outer part. A few other galaxies show a small region near the center that rotates in the same direction as the rest of the galaxy, but much faster. Either way, the part near the center that shows a strange pattern of rotation (the "core") has been interpreted as a distinct dynamical subsystem. Very briefly, the observational data seem to be that (1) anomalies show up in rotation curves near the centers of some elliptical galaxies and that (2) galaxies with these strange rotational properties do not show a photometric signature: there are no noticeable bumps in the brightness profile and no unusual shapes of isophotal contours that would suggest an excess of matter concentrated near the center. No strong color variations have been reported. The puzzle is to learn what we can about elliptical galaxies in general, and about galaxies with strange central regions in particular, from these observation:l facts.

Our approach is experimental. We make a guess about the form of the dynamically distinct subsystem, and then build a galaxy model to test experimental consequences such as the amount of matter required to produce observable effects and the length of time over which these effects would remain observable. We sidestep questions how the galaxy might have gotten to be that way in the first place. That gives us more freedom to explore a va riety of suggestions about what kind of dynamical system might give rise to the observed rotational patterns.

\section{Experiments with a Core}

The first experiment was to put a core inside an otherwise unexceptional elliptical galaxy model. The galaxy was oblate and axisymmetrical, consisting of 100,000 particles, and a core consisting of 1024 particles was placed at rest near its center. Some rotation encouraged the main galaxy to remain oblate and axisymmetric. The core initially occupied a spherical region more or less the size of the galaxy's core radius, and velocity dispersions of core particles were low enough that the core did not disperse. In addition, the core could be made to rotate in some arbitrary direction defined by an angle, $\theta$, measured from the galaxy's symmetry axis $=$ the axis of angular momentum $=$ the $z$-axis. Core particles are 
identical to galaxy particles from a dynamical point of view; they are marked to facilitate making movies and for later analysis.

Several experiments were run with this configuration, with differing amounts and directions of initial core rotation. The first control experiment with core angular momentum antiparallel to the galaxy's symmetry axis $\left(\theta=180^{\circ}\right)$ showed nothing unusual. The angular momentum remained in that direction and did not change.

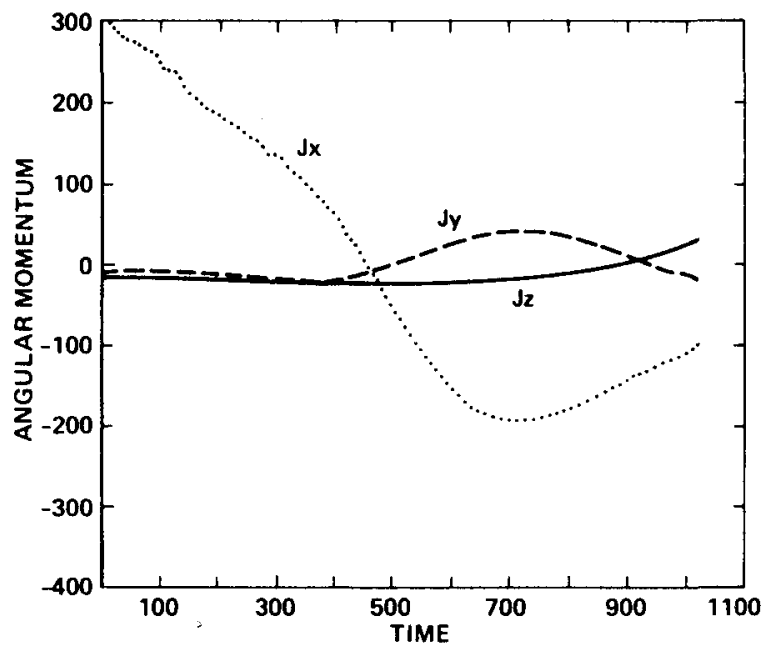

Figure 1. Components of total core angular momentum in an experiment with initial core angular momentum in the $x$-direction $\left(\theta=90^{\circ}\right) . J_{x}$ goes through zero and becomes negative. It damps out at long times, and it tends toward a state of no core rotation.

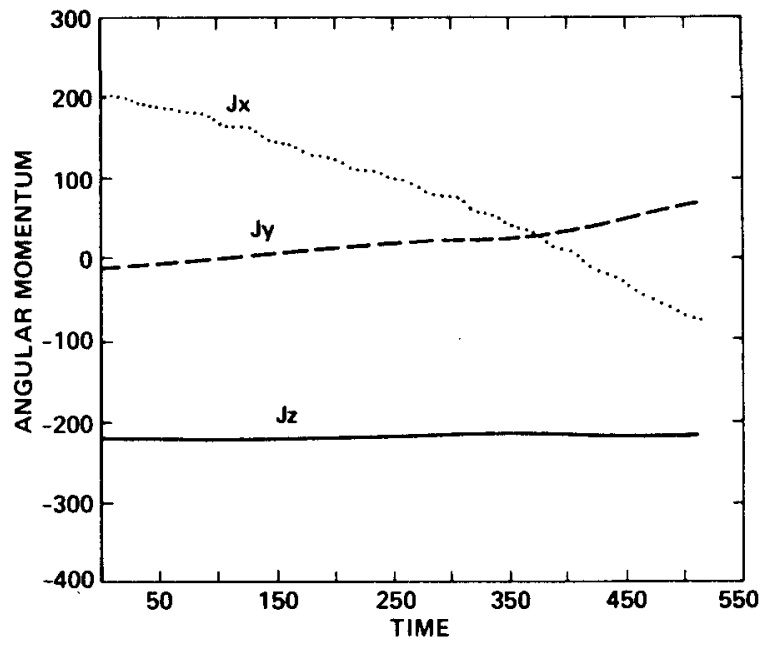

Figure 2. Components of total core angular momentum in an experiment with initial core angular momentum at $\theta=135^{\circ}$. $J_{x}$ diminishes as before, while $J_{z}$ remains nearly constant, ultimately leading to a counterrotating core. The time scale differs from that of Figure 1.

Experiments in which the core angular momentum lay in other directions were more interesting. Results are most easily summarized by means of plots of the core angular mo mentum as functions of the time. Results from an experiment with core rotation perpendicular to the galaxy's rotation $\left(\theta=90^{\circ}\right)$ are shown in Figure 1. All three components of core angular momentum are shown. The $x$-component decreases through zero, swings negative, and starts again toward zero, in the manner of a slow damped oscillation. The slow damping of perpendicular components of angular momentum appears to be a phase-mixing effect caused by anharmonicity of the galaxy's gravitational potential down near the center. The $y$ - and $z$-components drift a bit at late times. We return to that shortly. Results from an experiment with $\theta=135^{\circ}$ are shown in Figure 2. Again, the component perpendicular to the galaxy's symmetry axis oscillates slowly and shows signs of damping. 


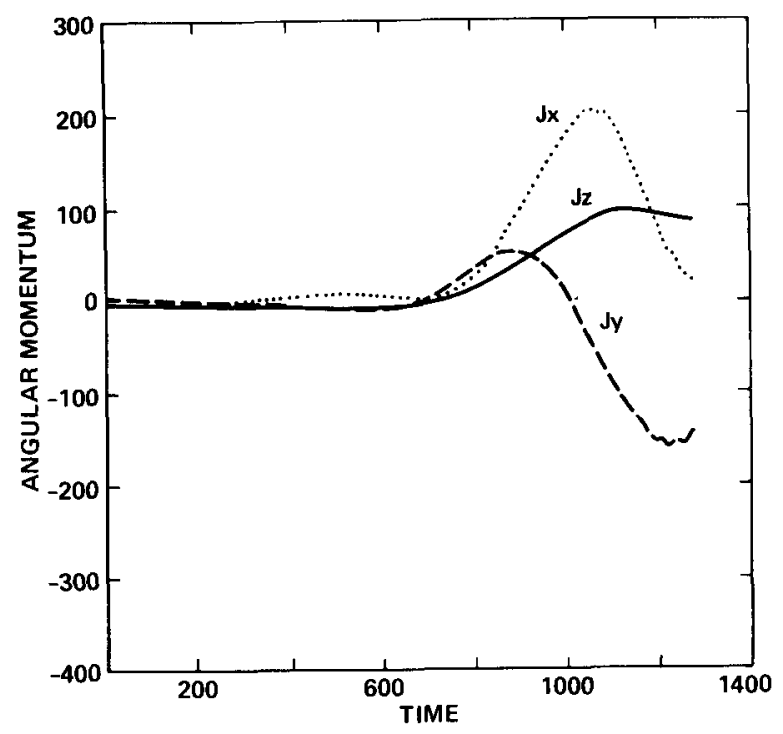

Figure 3. Components of total core angular momentum in an experiment with no initial core angular momentum. Growth of angular momentum at late times was found to result from slow disintegration of the core as it developed an orbiting motion around the center of the galaxy.

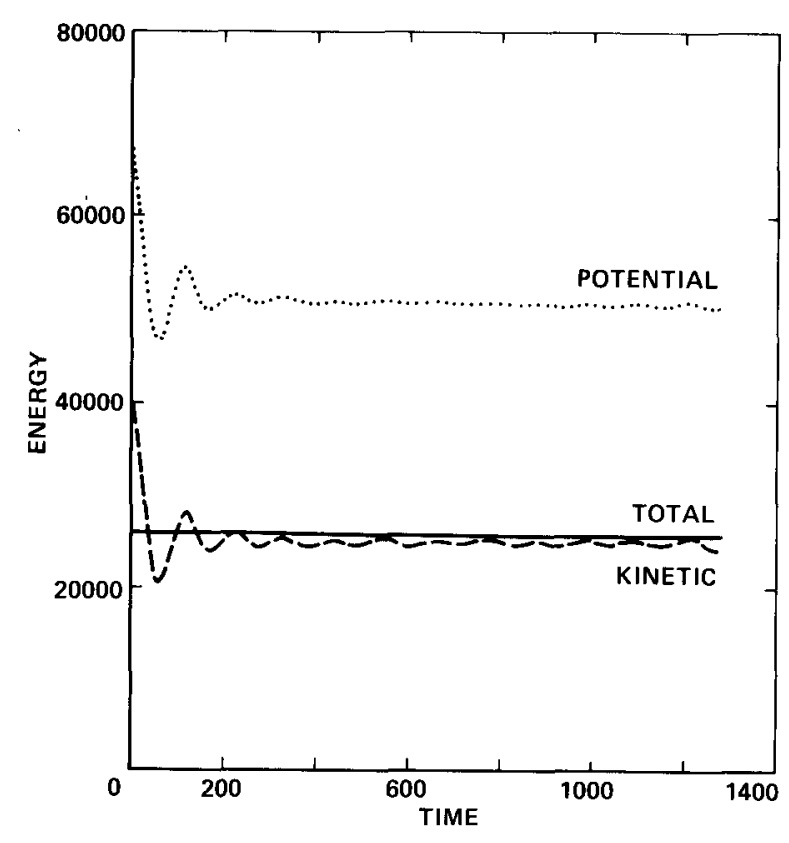

Figure 4. Total potential energy (dotted curve), total kinetic energy (dashed), and total energy as a function of the time. An initial lack of balance damps out quickly so most of the experiment runs in a very quiet galaxy ("virialized").

The drifts in angular momentum components that started out to be zero, as seen in Figures 1 and 2, invite further checks. We had to understand what causes them. They are, allowed by the physics, since the core can exchange angular momentum with the parent galaxy. If they are real, they might suggest a way to generate rotation within a core. We checked this with an experiment in which the core angular momentum was initially zero (Figure 3). This experiment ran quite a bit longer than the others, and core angular momentum built up appreciably. Again the perpendicular components oscillate slowly while the $z$-component appears to drift. Plots of the total potential and total kinetic energy as a function of time in Figure 4 show that the galaxy is very quiet long before the angular momentum drifts appreciably.

A check to find out why the core angular momentum was drifting led back to a phenomenon we've reported before-the core starts to orbit around the galaxy center (Miller and Smith 1990a). The buildup of orbital motion is shown in Figure 5. Orbital motion is much faster than the slow oscillation period associated with core angular momentum drifts. The core takes a beating as a result of this orbiting-it builds a tail and it takes on a shape something like a comet. At this stage, corrections to take out core velocity and position in angular momentum estimates become tricky. The core is no longer a nice compact object. 
Various parts are going in various directions. When angular momentum is computed relative to the center of mass for such an object, the result need not make a lot of sense. The drifts in Figures 1-3 are caused by this effect.

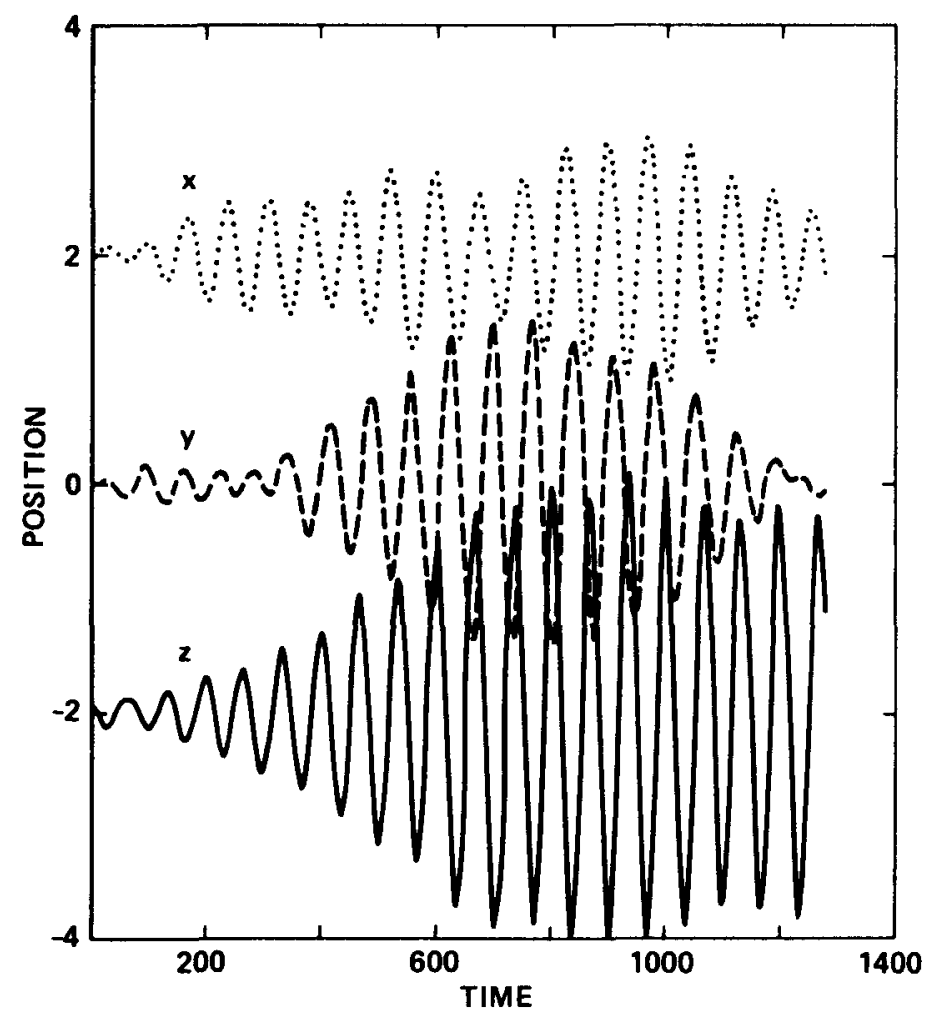

Figure 5. Position of the core's center of mass as a function of time in an experiment with no initial core angular momentum. The $x-$ and $z$-components have been displaced vertically for clarity; all three had zero mean displacements. The entire core starts to orbit.

One unit of time is about 3 million years in all these plots. A crossing time is about 100 million years or 35 units of time in the plots. The period of the slow oscillation in $J_{x}$ is given by the difference of oscillation frequencies for a particle near the center to oscillat. parallel to the equatorial plane or along the symmetry axis. A quarter cycle is about 500 time units or 1.5 billion years for the relatively round galaxies used here, and the damping due to phase mixing takes about 5 or 6 billion years. $J_{x}$ oscillates faster for flatter configu rations, but the phase-mixing damping rate appears to be about the same.

Density profiles were not affected noticeably in these experiments because the core has so little mass. We can get by with such a small core mass because we can study core rotation without the observer's signal-to-noise problem, but it is clear that quite a bit of mass would have to be included for the core to dominate the spectroscopic signature from the central region of a real galaxy. Further, speeds in the core's orbital motion are comparable to rotational speeds within the core. The observations seldom show translational motion of the core nearly as large as rotational velocities, so this doesn't seem to be a very good way 
to build counter-rotating cores. Slow disintegration of the core, associated with its orbital motions, also makes a distinct core seem an unlikely candidate to produce the observed counter-rotation effect.

\section{A Sheet}

A second possible configuration that might give rise to something that would look like a counter-rotating core is an apparent counter-streaming flow in a warped disk as seen from certain view directions. Imagine a sheet with a hat-brim warp and with a streaming flow in the same sense everywhere on the sheet. Imagine that you view this sheet from a direction nearly "face-on," but in which the inner portion of the sheet is inclined in one direction and the outer portions in the other direction relative to your view. You will then see one sense of rotation indicated by radial velocities in the inner portion, the opposite sense in the outer portions. Apparent changes of radial velocity become much more pronounced if the central portion of the disk is viewed nearly edge on, although this requires a fairly strong warp to yield reasonably large velocities in apparent counter-rotations. This effect is apparent in the warped disk of one of our recent studies (Miller and Smith 1990h). The long life of such a disk makes this an attractive picture, but strong counter-rotation is observable only from a fairly narrow range of viewing directions. Another problem with this picture is that most of the signal comes from the central portions-there is very poor signal-to-noise in the outer portions. Nonetheless, one can easily imagine that a small disk of material near the center of the galaxy, precessing within the main potential field, will show apparent rotation in unexpected directions, and might very well provide a reasonable model for counter-rotating cores.

\section{Remarks}

Many other configurations can be imagined that might look like counter-rotating cores from certain view directions. The two reported here are only those that we have tried so far. Any of these pictures has several problems. But any suggestion must be checked dy. namically for surprises like the core oscillations shown in Figure 5.

One problem in any of these pictures is to get enough stuff to make anomalous velocities show up observationally without having a photometric signature.

\section{References}

Bender, R. 1988, Astr.Ap., 202, L5.

Franx, M., and Illingworth, G. 1988, Ap.J.(Letters) 327, L55, Fiche 61-C2.

Illingworth, G. D., and Franx, M. 1989, In Dynamics of Dense Stellar Systems, Proceedings of a Workshop held at the University of Toronto, May 27-28 1988, Ed. D. Merritt (Cambridge: Cambridge University Press), pp. 13-23.

Jedrzejewski, R., and Schechter, P. L. 1988, Ap.J.(Letters) 330, L87, Fiche 104-F1. Miller, R. H., and Smith, B. F. 1990a, "Off-Center Nuclei in Galaxies" (submitted). Miller, R. H., and Smith, B. F. 1990b, "An Experimental Study of Disks in Axisymmetric Galaxies" (submitted). 


\section{DISCUSSION}

Galletta: About the possibility that box orbits are present in the galaxy: significant rotation in galaxies with counterrotating cores is an indication that box orbits are not dominant, since they contribute only to the velocity dispersion and not to the mean rotation.

Miller: That's the conventional picture. Numerical experiments show oscillations within a galaxy at a level of 2-58 of local density. Both the potential field and orbits of individual stars partake of this oscillation as well. Orbits that would be near the boundary that separates box and tube orbits in a static mean potential will diffuse across the boundary in a real galaxy. These are recently discovered effects whose importance is not yet known, but it seems likely that the distinction between box and tube orbits may become blurred. These oscillations may be thought of as the analog of solar oscillations in a galaxy.

Roos: Did you check whether the amplitude of the oscillations depends on parameters like the number of particles?

Miller: We checked many such things, as described in a paper that is in the reviewing process. Amplitudes vary inversely as the square root of particle number, but the growth rate is independent of particle number. That is more important. $\sqrt{\mathrm{N}}$ dependence many sound artificial, but the real artificiality in $n$-body work is that we start the nucleus atop the mass centroid, something that could never happen in a real galaxy.

Bland: (Here's an opportunity for theoreticians to exonerate themselves.) Van Albada showed in the 50's that violent relaxation in collapsing systems gives rise to an $r^{1 / 4}$ law in the light profile. Given Gillian Wright's beautiful photometry on Arp 220, do numerical simulation still show the $r^{1 / 4}$ law as an eventual consequence.

Norman: T. van Albada (1982) showed that you only get an $\mathrm{r}^{1 / 4}$ law from cold collapse systems.

Miller: Most $n$-body systems that have been shaken vigorously enough, whether by merger, fairly close collisions, or tidal stretching as the galaxy orbits near the center of a galaxy cluster, reshuffle their mass into a distribution that projects to a de Vaucouleurs profile. But the observed profile is a light profile, so association of this n-body result with the observed result requires that the light trace the mass faithfully, that the system be mixed to have constant $M / L$ everywhere. Since preencounter galaxies are thought to have different $M / L$ in different places, mixing is required. That is likely, and we all hope it's true, but mixing has not been demonstrated as the mass gets redistributed. 